\title{
Sandhi externe en macédonien et en roumain. Les assimilations de sonorité
}

\section{External sandhi in Macedonian and Romanian. The voicing assimilations}

\author{
Anna Cychnerska \\ Uniwersytet Mikołaja Kopernika w Toruniu \\ anna.cychnerska@umk.pl
}

\begin{abstract}
The paper presents a comparative analysis of the assimilation in terms of voicing on the border between words in Macedonian and in Romanian. Although the compared languages belong to different language families, the pronunciation of the obstruental segments in this position is similar. In both Romanian and Macedonian, a regular regressive assimilation is observed on the border between two words. In Macedonian, the voiced obstruent at the end of a word can be pronounced as a voiced or a voiceless before a resonant at the beginning of the next word. It is important that more often the voiced obstruent at the end of the word loses voicing before a vowel in the initial position than before a sonant. In Romanian, before a resonant in the initial position, the voiced obstruent in the final position of the word in front of it is pronounced voiced. The voiceless obstruent on the border between words can be pronounced as a voiced if it occurs before a sonant in the initial position of the next word. This is an optional phenomenon like the voiceless pronunciation of the voiced obstruent before a resonant on the border of two words in Macedonian. As one can see, in both languages, obstruents at the end of a word behave differently before initial vowels and sonants on the inter-word border.
\end{abstract}

Keywords: sandhi, voiced obstruent, voiceless obstruent, vowel, sonant, assimilation, inter-word boundary, Macedonian, Romanian

Les langues slaves se caractérisent par une grande variété de façons dont elles réalisent la sonorité à la frontière de deux mots phonétiques en contact. Dans la plupart de ces langues (à l'exception du serbe, du croate et de l'ukrainien) ${ }^{1}$, ce sont

\footnotetext{
${ }^{1}$ Dans les langues serbe et croate, les assimilations de sonorité n'ont pas lieu entre deux mots accentués adjacents. Cela se réfère autant aux enchainements d'obstruantes qu'aux réalisations d'une
} 
les assimilations de sonorité régressives qui se produisent dans les groupes d'obstruantes entre les mots. En outre, ces langues diffèrent dans la façon dont l'obstruante finale est réalisée avant une résonnante initiale du mot qui suit, ce qui constitue les bases de la distinction d'un sandhi externe assourdissant (en russe, biélorusse, bulgare, slovène, tchèque, et dans la variante nord-est du polonais) et d'un sandhi externe sonorisant (en slovaque et dans la variante sud-ouest du polonais) (cf. Sawicka, 2001).

Prises en compte la régularité des assimilations de sonorité dans les enchaînements d'obstruantes et l'absence de réaction de la part des obstruantes finales aux résonnantes initiales entre deux mots en contact, les premières descriptions de la phonétique macédonienne relatives aux phénomènes se produisant entre les mots classifiaient cette langue comme un type intermédiaire entre le groupe de langues dans lesquelles le sandhi atteint son étendue entière et le groupe de langues qui ne connaissent pas le sandhi (cf. Конески, 1996 ; Савицка, Спасов, 1991). Les études récentes sur le macédonien moderne ont démontré que dans la position discutée, les consonnes sonores proprement dites peuvent garder leur sonorité ou la perdre avant les résonnantes initiales (Sawicka, 2003 ; Korytowska, 2012).

Contrairement au macédonien, le roumain n'a été, jusqu'aujourd'hui, l'objet d'aucune recherche approfondie concernant la sonorité, telle qui se base sur l'analyse du matériel enregistré. La littérature du domaine ne contient que des données rudimentaires sur la façon dont sont réalisés les segments consonantiques dans des contextes phonétiques sélectionnés. Les descriptions faisant partie de cette littérature ne rendent pas compte des règles précises du sandhi, et soulignent souvent qu'une façon de prononcer analysée est une variante qui dépend également de facteurs extra-phonétiques (cf. Turculeţ, 2016, pp. 220-223).

Des informations rares sur la réalisation de la sonorité aux frontières des mots peuvent être trouvées uniquement dans une monographie et quelques articles d'Andrei Avram. L'auteur caractérise les assimilations de sonorité régressives entre deux unités accentuées en roumain comme fréquentes, mais facultatives. L'assimilation peut se produire dans les enchaînements d'obstruantes - quand ils sont formés par des segments étymologiquement hétérogènes du point de vue de leur sonorité ainsi que dans des groupes tels que « une obstruante sourde + une sonante », où l'on observe le voisement facultatif du premier élément du groupe (cf. Avram, 1961 ; 1986, pp. 551-574; 2014, pp. 162-178).

Le but de cet article est de comparer les assimilations de sonorité dans le cadre du sandhi externe en macédonien et en roumain. Il semble qu'en dépit des

\footnotetext{
obstruante finale avant une résonnante initiale ; dans ce contexte, la qualité de la consonne obstruante ne devrait pas être modifiée. Dans l'enchaînement des mots en ukrainien, le voisement régressif qui a lieu dans les grupes d'obstruantes - dont le premier élément est sourd - appartient à la norme. D'autre part, l'obstruante finale maintient sa sonorité avant une obstruante sourde, de même qu'avant une résonnante, à l'initiale.
} 
différences génétiques, ces langues se caractérisent par certains traits communs, bien que d'autre part - ce qui est évident - elles conservent également leurs caractéristiques spécifiques. Dans l'article, j'utilise en partie les résultats de ma propre analyse d'enregistrements macédoniens antérieurement effectuée, dont j'ai choisi ceux qui se réfèrent aux enchaînements entre les mots (Korytowska, 2012). Pour le roumain, le matériel source est un livre audio intitulé « Oscar et la Dame rose » par Éric-Emmanuel Schmitt. Le texte enregistré au format CD audio a été lu par l'acteur Marius Manole. La durée de l'enregistrement est d'environ 82 minutes. Il a été écouté trois fois. Certains contextes, en plus, ont été présentés pour l'écoute aussi à une autre personne - spécialiste de formation phonétique.

Environ 780 enchaînements entre les mots ont été sélectionnés en vue de les vérifier à l'écoute. Eu égard au type de segments dont ces enchaînements sont formés, ils peuvent être divisés en quatre groupes:

- le $\mathrm{I}^{\mathrm{er}}$ groupe : une obstruante finale sourde + une obstruante initiale sonore,

- le II ${ }^{\mathrm{e}}$ groupe : une obstruante finale sonore + une obstruante initiale sourde,

- le III ${ }^{\mathrm{e}}$ groupe : une obstruante finale sonore + une résonnante initiale (prise en compte la différenciation des positions avant une voyelle et avant une sonante à l'initiale),

- IV ${ }^{\mathrm{e}}$ groupe : une obstruante finale sourde + une résonnante initiale (prise en compte la différenciation des positions avant une voyelle et avant une sonante à l'initiale).

À côté des segments obstruants simples, l'étude se concentre sur les groupes consonantiques, y compris les groupes formés d'une sonante et d'une obstruante. À l'initiale du mot, aussi les diphtongues ont été prises en considération.

Pour illustrer les contextes des enchaînements entre les mots, des spectrogrammes et des oscillogrammes ont été préparés à l'aide du logiciel Praat (version 6.1.05), dont certains seront présentés plus loin. L'étendue de l'échantillon choisi pour étudier le sandhi externe en roumain fait considérer cette recherche comme introductive.

Comme cela a été déjà mentionné, les assimilations de sonorité en macédonien qui touchent les groupes d'obstruantes entre les mots doivent être considérées comme très régulières. La façon de réaliser les enchaînements consonantiques est fortement influencée par le tempo de la parole. À un tempo rapide, l'adhésion des éléments constituant l'enchaînement de deux mots est forte, ce qui favorise les assimilations. Un tempo plus lent peut affaiblir l'influence exercée par le second segment du groupe sur la consonne précédente, ce qui entraîne l'absence de l'assimilation (l'articulation du second segment commence après que le retentissement du segment précédent cesse), на вредно[s z]атоа, ко[ks d] одаде.

Le matériel analysé est dominé par les réalisations dans lesquelles l'assimilation de sonorité s'accomplit. Ce phénomène a été observé dans environ $99 \%$ des exemples consistant en l'enchaînement «une obstruante finale sonore + une 
obstruante finale sonore » et dans environ $82 \%$ des exemples consistant en l'enchaînement des obstruantes dans l'ordre inverse, par exemple гpa $[\mathrm{t}$ p] jazoдume, дру[k p]ат, сове[d z]a петок, конкурсо[·d]енес, ja[z b]ев, факто[ d]ека (voir fig. 1). Dans certains contextes analysés, l'apparition du support vocalique après le premier élément du groupe a empêché l'assimilation (p.ex. ше $\left.\mathrm{s}^{ } \mathrm{z}\right]$ апирка, конце $\left[\mathrm{pt}^{ } \mathrm{z}\right]$ а својствата, voir fig. 2); dans d'autres - cette assimilation ne s'est pas produite à la suite de l'élision du segment final : од фондот за здавството $\rightarrow$ од фонд[о з]а здравството.

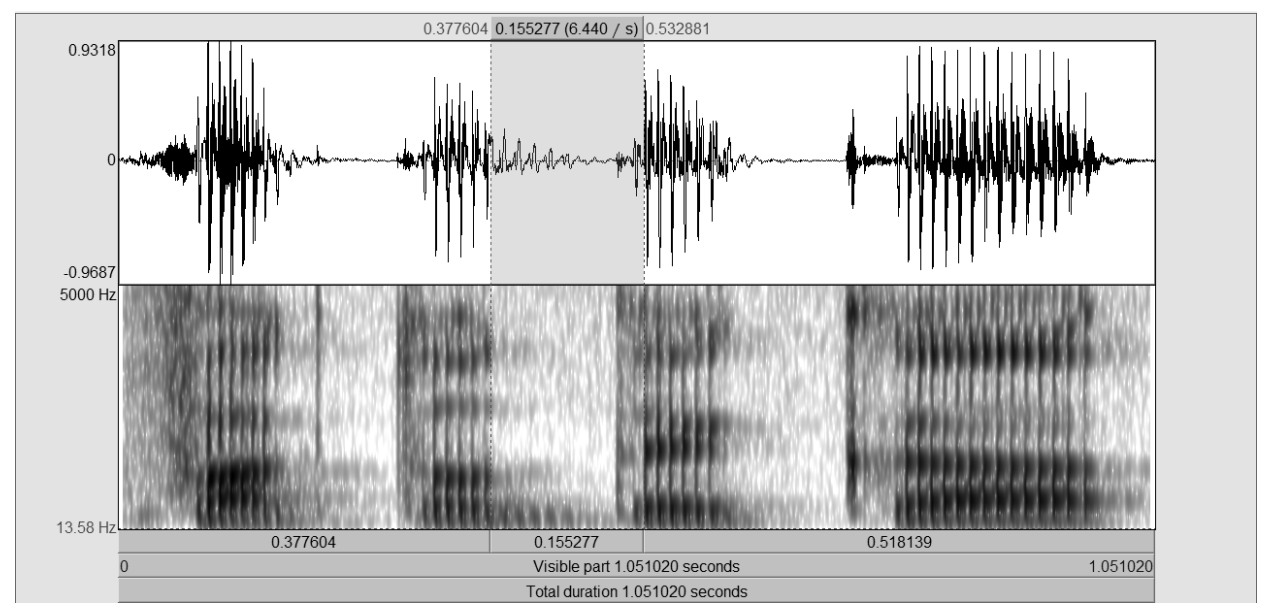

Figure 1. La réalisation voisée d'une obstruante finale sourde avant une obstruante initiale sonore - dans l'exemple фактот дека (une seule occlusion)

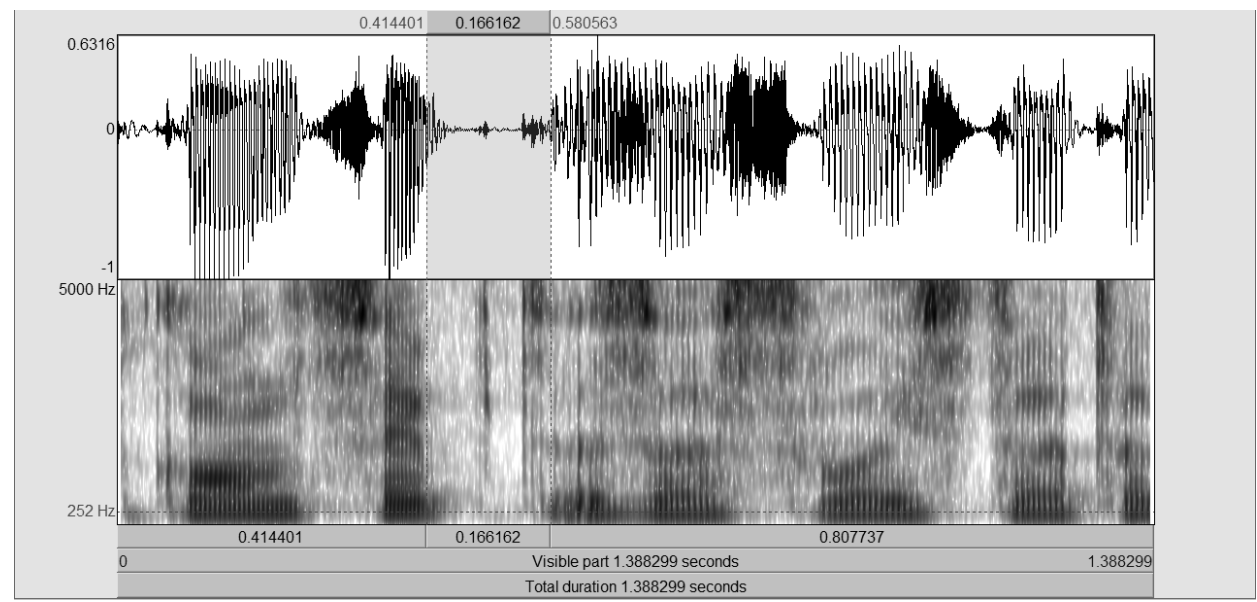

Figure 2. La réalisation sourde du groupe d'obstruantes final avant une obstruante sonore initiale - dans l'exemple кониепт за својствата 
La réduction des groupes consonantiques entre les mots est également fréquente en roumain. À l'élision sont souvent soumis : (1) le deuxième segment du groupe final « une sonante + une obstruante » avant une obstruante à l'initiale du mot qui suit (p. ex. coborâ[[n sk]ara, auzi[n tg]e auzisem, me[r s]ă mă odihnesc, fu[n p]e pat, aco $[r \mathrm{~s}] \breve{a}-m i$ faci - l'élision du /d/ dans les mots coborând, auzind, fund, acord, et celle $\mathrm{du} / \mathrm{g} /$ dans le mot merg), (2) l'obstruante finale postvocalique toute seule dans le même contexte (p. ex. un pi[ d]e fler - l'élision du /k/ dans le mot pic; afla $[\mathrm{v}] e s ̧ t i$ - l'élision du /t/dans le mot aflat; simţi[ b] ine - l'élision du /t/ dans le mot simţit $)^{2}$.

La prononciation dans le matériel analysé est dominée par les assimilations de sonorité régressives dans les enchaînements d'obstruantes entre les mots, p.ex. profi $[\cdot \mathrm{d}]$ e situaţie, purta $[\mathrm{d} \mathrm{b}]$ ine, porecli $[\mathrm{d} 3]$ umară, să-[3 b]ată, ro $[\mathrm{s} \mathrm{k}]$ are, cân $[\mathrm{t} \mathrm{f}] a c$, imagine [s p]e prietena, cre [t k]ă a murit (voir fig. 3 et 4). Dans le cas des enchaînements d'une affriquée finale et d'une occlusive ou obstruante fricative initiale, accompagnés de l'assimilation de sonorité, le premier segment du groupe consonantique est souvent fricatisé (voir fig. 5). Dans les enregistrements macédoniens, on ne constate pas des phénomènes pareils.

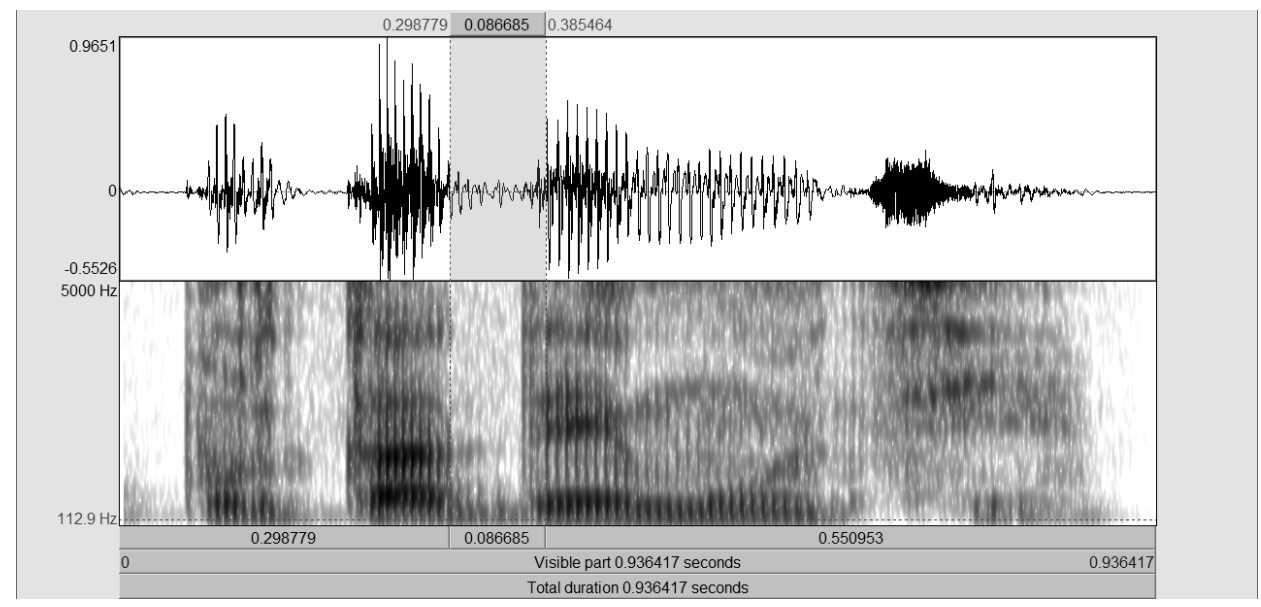

Figure 3. La réalisation voisée d'une obstruante sourde finale avant une obstruante sonore initiale - dans l'exemple putat de emoţie (une seule occlusion)

\footnotetext{
${ }^{2}$ Dans le groupe final - $n d$, c'est le segment $d$ qui est soumis plus souvent à l'élision dans la position avant une résonnante initiale.
} 


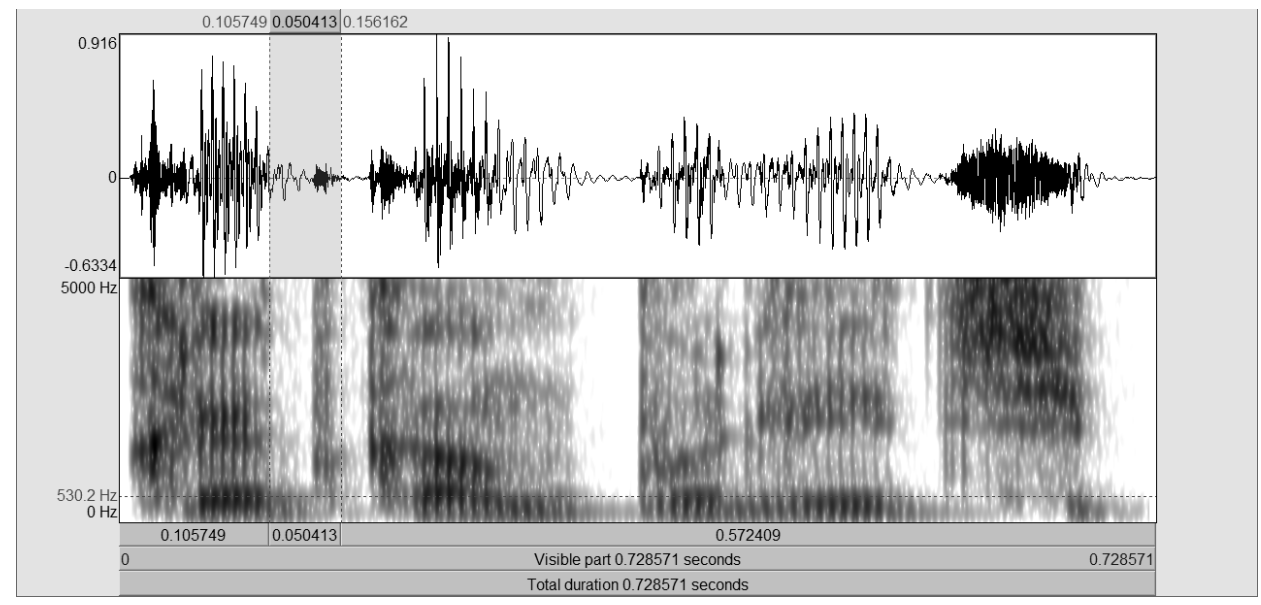

Figure 4. La réalisation dévoisée d'une obstruante finale sonore avant une obstruante initiale sourde dans l'exemple cred că am pârlit şi...

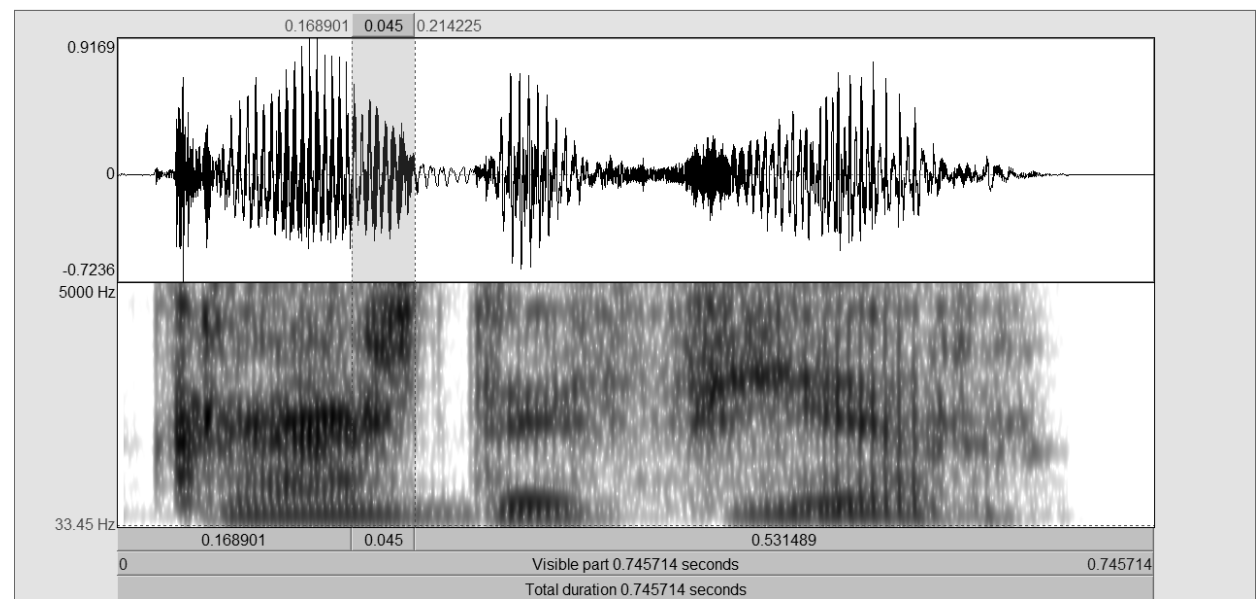

Figure 5. La réalisation voisée d'une obstruante finale sourde avant une obstruante initiale sonore - dans l'exemple craci de fier

Les réalisations sans assimilation dans le matériel roumain sont d'environ $10 \%$ ( $4 \%$ dans le cas du contexte « une obstruante finale sonore + une obstruante initiale sourde » et 12,5\% dans le cas du contexte dans l'ordre inverse, voir tab. 1). 
Tableau 1. La prononciation des enchaînements entre les mots sans assimilation de sonorité

\begin{tabular}{|c|c|}
\hline Exemple analysé & La façon de réaliser l'enchaînement entre les mots \\
\hline zic vreo & $z i[\mathrm{k} \mathrm{v}] r e o$ \\
\hline aşverea & $a\left[\int \mathrm{v}\right] r e a$ \\
\hline povestit vieţile & povesti $[\mathrm{t} \mathrm{v}]$ ieţile \\
\hline distrus viaţa & distru $[\mathrm{s}$ f $]$ iaţa \\
\hline nici viaţa & $\left.n i\left[\mathrm{y}^{\mathfrak{j}} \mathrm{v}\right]^{\mathrm{j}}\right]$ iaţa \\
\hline daţi veşti & $d a[\mathrm{ts} \mathrm{v}] e s ̧ t i$ \\
\hline ascultat valsul & asculta[t f]alsul \\
\hline Sarah Zvârlugă & Sara $[\mathrm{x}$ sv] $\operatorname{arlugă}(2 \mathrm{x})$ \\
\hline implinit zece ani & $\hat{\imath}$ implini $[\mathrm{t} \mathrm{s}]$ ece ani \\
\hline îti dai seama & $\hat{\imath}[$ ts t $]$ ai seama \\
\hline mică şi de slabă & mică $\left[\int \mathrm{d}\right] e$ slabă \\
\hline os de peşte & $o[\mathrm{~s} \mathrm{~d}]$ e peşte \\
\hline băț de slab & $b \breve{a}[$ ts d]e slab \\
\hline brusc de mine & bru[s: d]e mine \\
\hline glasate şi de şampanie & glasate [J: $\mathrm{d}]$ e şampanie \\
\hline iubeşti de fapt & iube $\left[\int \mathrm{t}\right]$ e fapt \\
\hline iubesc din tot sufletul & iube[s t] in tot sufletul \\
\hline mulţumesc doamne & mulţume $[\mathrm{s} \mathrm{t}]$ oamne \\
\hline văd pe Tanti Roz & vă[d p]e Tanti Roz \\
\hline bolnav sunt & bolna[v s]unt \\
\hline grav pentru & gra $[\mathrm{v}$ p]entru \\
\hline
\end{tabular}

Comme il résulte du tableau ci-dessus, dans les exemples où les assimilations de sonorité n'ont pas lieu dans la prononciation de l'acteur roumain, l'un des éléments $\mathrm{du}$ groupe consonantique est l'obstruante /v/. Dans l'enregistrement, il y a au total 13 enchaînements du type « une obstruante sourde $+/ \mathrm{v} /$ » (7 d'entre ceux-ci sont réalisés sans assimilation, voir fig. 6,7) et 4 enchaînements dans l'ordre inverse (y compris 2 réalisations avec assimilation) $)^{3}$. Dans quelques exemples, on observe aussi un phénomne intéressant: l'assimilation progressive de sonorité. Cela concerne les enchaînements du type "une obstruante sourde + une obstruante sonore ». C'est l'assimilation regressive entre les segments constitutifs du groupe qui devrait se produire dans le contexte pareil conformément à la norme. Au lieu du voisement de la consonne finale donc, sous l'influence de la consonne qui suit, on constate dans la prononciation du lecteur roumain le dévoisement de la consonne initiale sonore.

\footnotetext{
${ }^{3}$ Dans le matériel analysé, j'ai découvert un exemple de l'enchaînement $« / \mathrm{v} /+/ \mathrm{d} / »$ dans lequel le segment final et l'obstruante initiale sont soumis au dévoisement : groza[f t $]$ e mândru.
} 


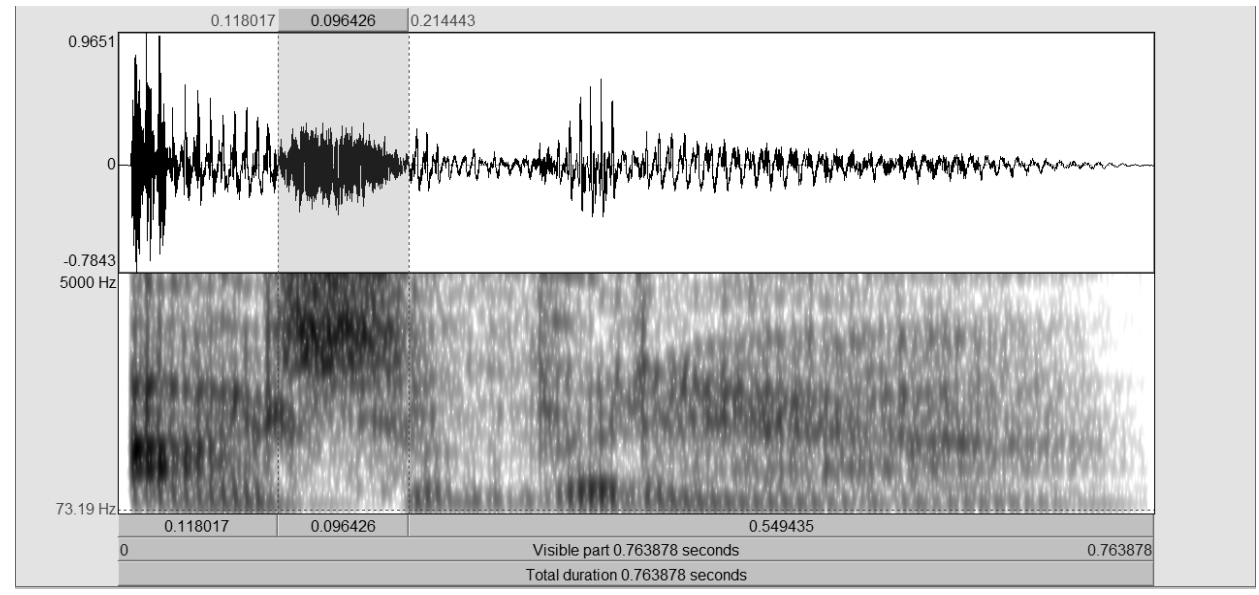

Figure 6. La réalisation sourde d'une obstruante finale sourde avant le /v/ initial - dans l'exemple aş vrea

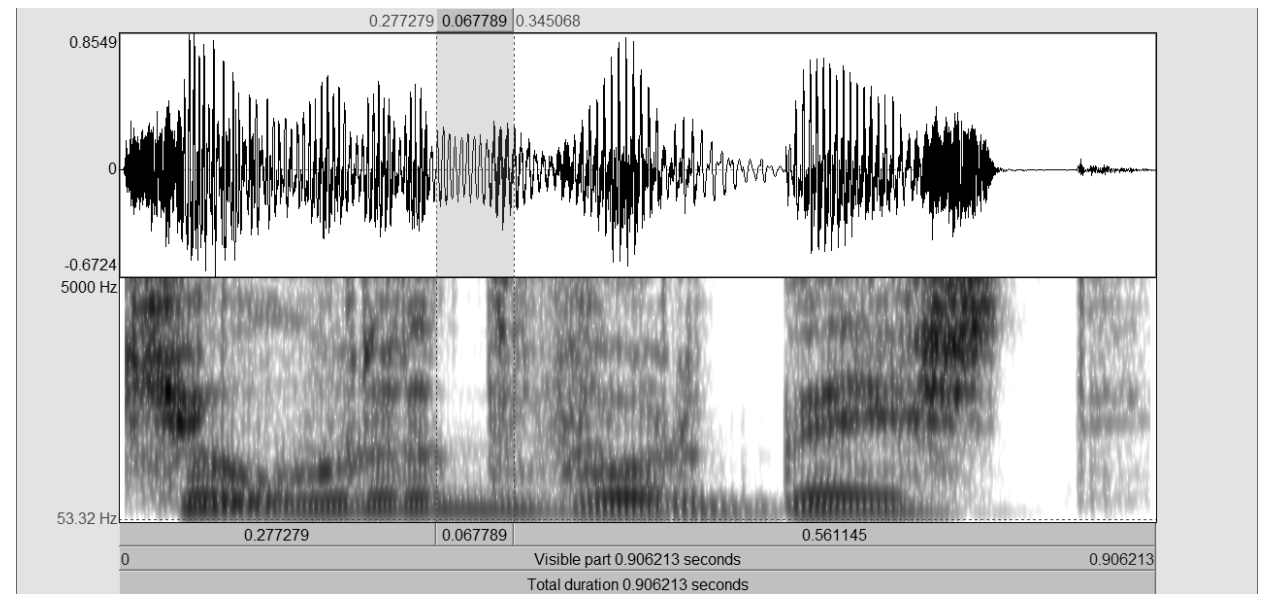

Figure 7. La réalisation voisée d'une obstruante finale sourde avant le /v/ initial - dans l'exemple ce urât vorbeşti

Conformément à la norme littéraire macédonienne, aux frontières des mots, tant les obstruantes sonores que les obstruantes sourdes devraient conserver leur qualité avant le /v/ initial de l'unité accentuée voisine, tandis que le /v/ final devrait être dévoisé avant une obstruante sourde initiale. Dans les enregistrements analysés, la prononciation des lecteurs a été conforme à la norme dans le cas des enchaînements $\mathrm{du}$ type « une obstruante finale sourde + le $/ \mathrm{v} /$ à l'initiale » et du type «le /v/ à la finale + une obstruante initiale sourde », p. ex. напредо[k v]о работата, на 


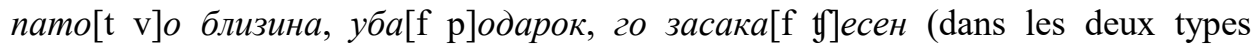
d'enchaînements, l'écart par rapport à la norme est inférieur à $0,5 \%$ ). J'ai découvert le dévoisement d'une obstruante sonore finale avant le $/ \mathrm{v} /$ initial dans environ $22 \%$ des exemples consistant en le contexte discuté, p. ex. $ә р у[\mathrm{k} \mathrm{v}] и д, ~ п р е в о[\mathrm{~s} \mathrm{v}] о$ градовите (voir fig. 8). Dans les autres exemples, les deux segments du groupe d'obstruantes ont eu la réalisation sonore, p. ex. гра $[\mathrm{d} \mathrm{v}]$ о Македонија, дру $[\mathrm{g} \mathrm{v}] и \partial^{4}$.

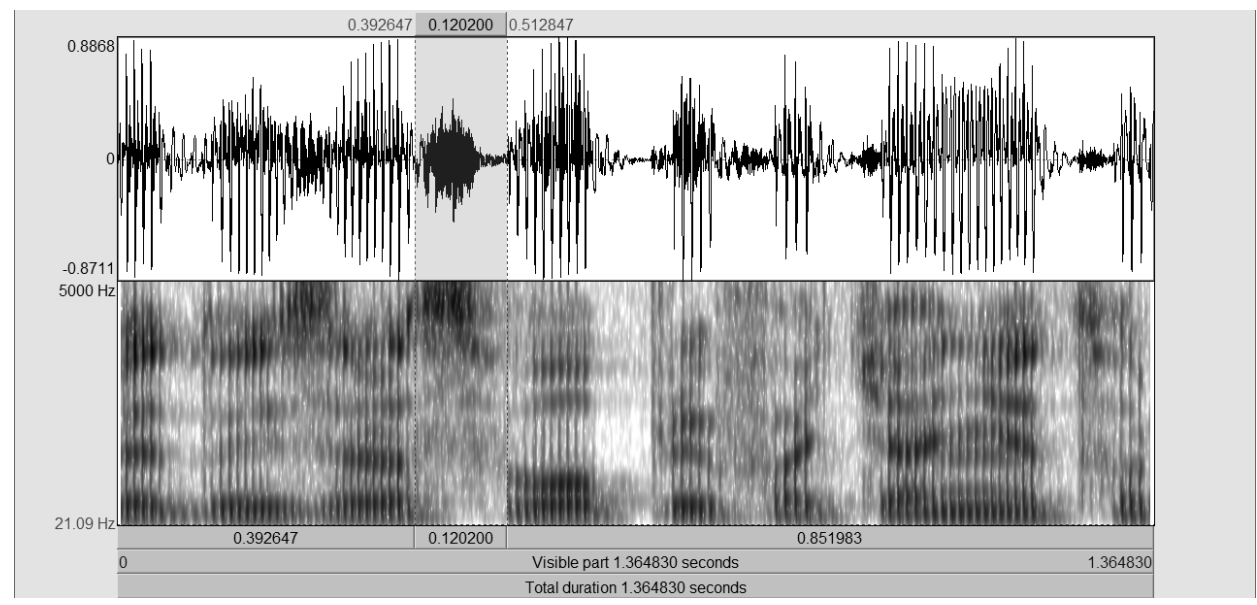

Figure 8. La réalisation dévoisée de l'obstruante sonore finale avant le /v/ initial - dans l'exemple до израз во прифаќањето

Pour les deux langues comparées, bien que dans des limites inégales, on peut proposer la thèse qu'il y a une différence dans l'influence des voyelles et des sonantes sur la façon de réaliser la sonorité à la frontière des mots en contact. La vérification de la réalisation des obstruantes sonores finales avant les résonnantes sonores en macédonien a démontré qu'aux frontières de deux unités accentuées en contact, les dévoisements facultatifs du premier segment de l'enchaînement se produisent plus souvent avant une voyelle initiale (environ $60 \%$ des réalisations dévoisées d'une obstruante sonore finale, p. ех. пора[s o]колу, Белгра[t i] Приитина) qu'avant une sonante initiale (environ $29 \%$ des réalisations pareilles, p. ex. на дру[k n]ачин, за изле[s n] адвор) (voir fig. 9 et 10).

\footnotetext{
${ }^{4}$ Pour en savoir plus sur la réalisation $\mathrm{du} / \mathrm{v} /$ dans la standard macédonien : Korytowska, 2012, pp. 201-238.
} 


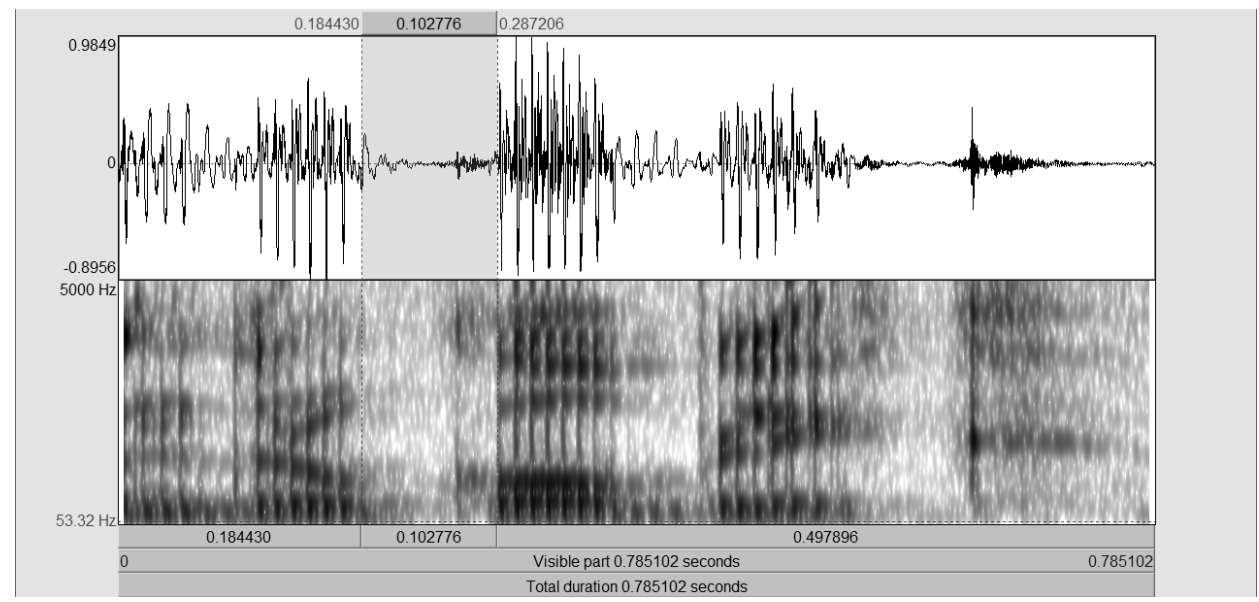

Figure 9. La réalisation dévoisée d'une obstruante finale avant une voyelle initiale - dans l'exemple друг облик

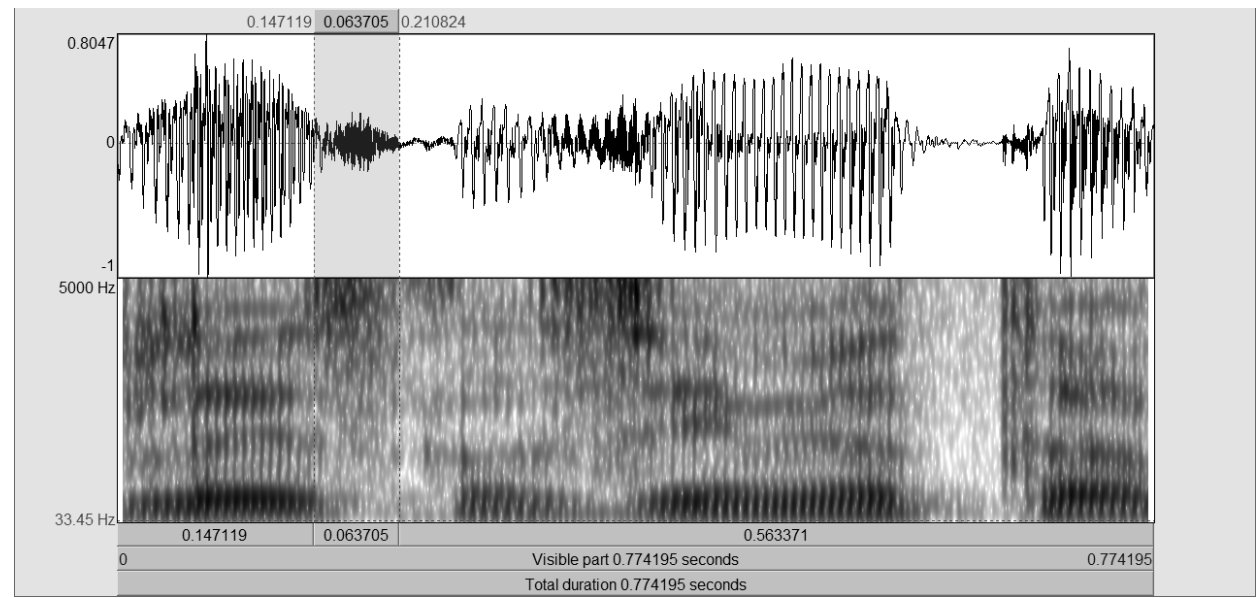

Figure 10. La réalisation dévoisée d'une obstruante sonore finale avant une sonante initiale - dans l'exemple влез на земјата

En roumain, la norme prévoit que dans les enchaînements entre les mots, les obstruantes sonores finales maintiennent leur sonorité avant une résonnante initiale. Dans le matériel enregistré soumis à l'analyse, chaque prononciation était conforme à cette norme. On observe cependant que le groupe final -nd est plus souvent exposé aux réductions avant une sonante (surtout nasale) qu'avant une voyelle (élision de /d/ dans $95 \%$ des exemples avec une sonante à l'initiale et dans environ $33 \%$ des 
exemples avec une voyelle à l'initiale). L'analyse a pris aussi en considération l'influence d'une résonnante initiale sur la façon de réaliser les obstruantes sourdes finales. Pendant qu'une voyelle initiale ne peut pas sonoriser une obstruante sourde finale, d'après A. Avram le voisement d'une obstruante sourde finale avant une sonante est facultatif. La vérification de ce contexte a confirmé l'hypothèse que les voyelles n'influencent pas l'obstruante sourde finale. Dans le cas des enchaînements d'une obstruante sourde finale et d'une sonante initiale, le voisement de la consonne proprement dite a été observé dans 22 exemples (environ 14,6\% de tous les groupes contenant ce contexte). Une prononciation de la consonne sourde finale dans l'enchaînement qui n'était pas accompagnée de changements de qualité dominait manifestement, p. ex. s-a uita[t l]a mine, $n-a$ spu[s n]imic, decâ[t m]ine (voir Fig. 11). Dans la plupart des exemples où le voisement s'est produit, les enchaînements consistaient en les groupes d'une affriquée sourde finale et d'une sonante nasale initiale. Dans ces réalisations, la fricatisation du segment obstruant accompagnait le dévoisement (voir fig. 12).

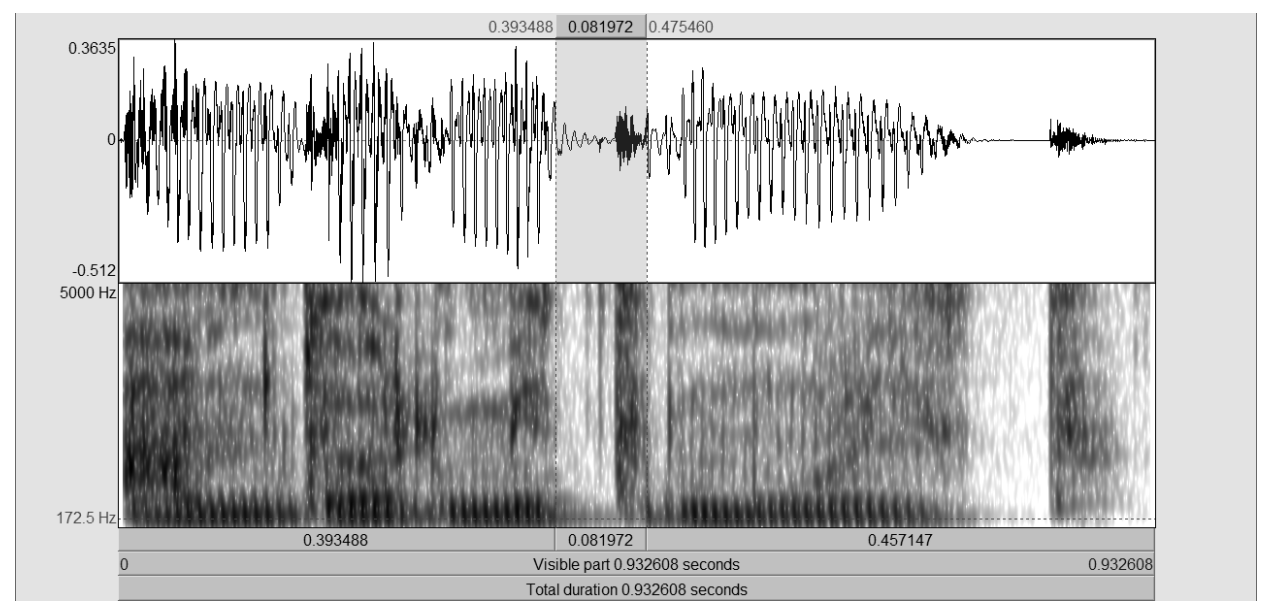

Figure 11. La réalisation sourde d'une obstruante sourde finale avant une sonante initiale - dans l'exemple am dormit mult 


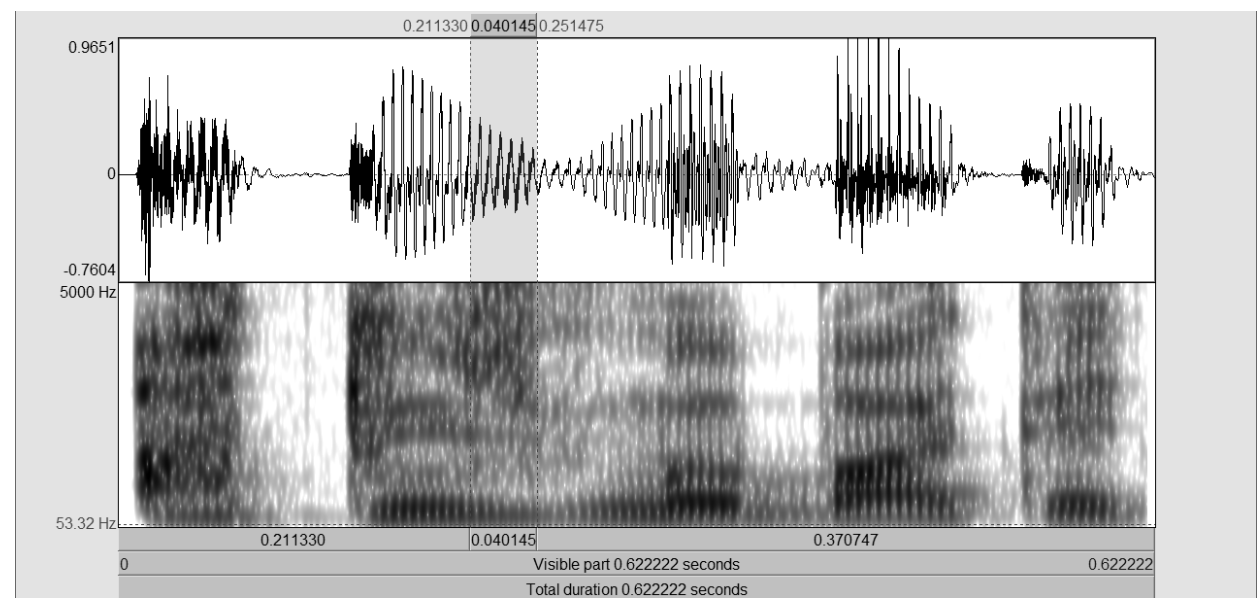

Figure 12. La réalisation voisée d'une obstruante sourde finale avant une sonante initiale - dans l'exemple atunci mă bata

Comme il résulte de l'analyse effectuée, il est possible de trouver certaines ressemblances entre les langues comparées en ce qui concerne la façon de réaliser la sonorité dans le cadre du sandhi externe. Tout d'abord, les assimilations de sonorité se produisent dans les deux langues assez régulièrement dans les groupes d'obstruantes aux frontières des mots en contact. Une absence rare de l'assimilation régressive dans ce contexte peut être observée aussi bien en macédonien qu'en roumain dans les enchaînements du type «une obstruante sourde finale + une obstruante sonore initiale » plus souvent que dans les enchaînements dans l'ordre inverse. Dans les deux langues, on peut observer un caractère instable des réalisations de sonorité dans des enchaînements, dont l'un des segments constitutifs est /v/.

Il existe des différences importantes entre le macédonien et le roumain en ce qui concerne la réalisation des obstruantes finales avant une résonnante initiale à la frontière des mots en contact. Dans le standard macédonien, on constate dans cette position les dévoisements facultatifs du premier segment du groupe, tandis qu'en roumain les obstruantes sonores conservent leur qualité sans exception. Dans la langue macédonienne, une voyelle ou une sonante à l'initiale ne provoquent pas le voisement d'une obstruante apparaissant à la finale du mot qui précède. En roumain, le voisement d'une obstruante sourde finale peut être causé uniquement par une sonante initiale du mot qui suit, bien que ce phénomène paraît assez rare. Malgré l'influence variée des résonnantes initiales sur la façon de réaliser les obstruantes finales, le fait important est que leur prononciation diffère ou bien peut différer avant une voyelle et une sonante dans les deux langues.

Je voudrais remercier ma collègue et amie, Dr Agata Trawińska (Cracovie) pour son aide apporté à l'écoute des enregistrements analysés et pour les consultations. 


\section{BIBLIOGRAPHIE}

Avram, A. (1961). Cercetări asupra sonorităţii în limba română. Bucureşti : Editura Academiei Române.

Avram, A. (1986). Sandhi phenomena in Romanian. In H. Andersen (ed.), Sandhi phenomena in the languages of Europe. Trends in linguistics. Studies and monographs, 33, pp. 551-574.

Avram, A. (2014). Studii de fonetică descriptivă (generală şi românească). Bucureşti : Editura Academiei Române.

Korytowska, A. (2012). Sandhi w standardzie języka macedońskiego. Toruń: Wydawnictwo Uniwersytetu Mikołaja Kopernika.

Sawicka, I. (2001). An Outline of the Phonetic Typology of the Slavic Languages. Torun: Wydawnictwo Uniwersytetu Mikołaja Kopernika.

Sawicka, I. (2003) Z problematyki sandhi w języku macedońskim. In W. Banaś, L. Bednarczuk, K. Polański (ed.). Études linguistiques romano-slaves offertes à Stanisław Karolak. Kraków: Edukacja, pp. 453-457.

Schmitt, E.-E. (2015). Oscar şi Tanti Roz. Dublu CD audio, lectura - Marius Manole. Bucureşti : Humanitas Multimedia.

Turculeţ, A. (2016). Introducere în fonetica generală şi românească. Iaşi : Casa Editorială Demiurg.

Конески, Б. (1996). Граматика на македонскиот јазик. Скопје: Детска радост.

Савицка, И., Спасов, Љ. (1991). Фонологија на современиот македонски стандарден јазик.

I. Скопје: Македонска академија на науките и уметностите. 